\title{
Influência da fertirrigação com água residuária sobre o solo e crescimento de mudas arbóreas
}

\author{
Influence of fertigation with wastewater on the soil and growth of tree seedlings \\ Influencia de la fertirrigación con aguas residuales en el suelo y crecimiento de plántulas de árboles
}

Recebido: 09/11/2021 | Revisado: 16/11/2021 | Aceito: 05/02/2022 | Publicado: 06/02/2022

Lucas Rafael Sanches

ORCID: https://orcid.org/0000-0002-5794-6281 Cooperativa Agroindustrial C. Vale, Brasil

E-mail: lucasrafaelsanchez@hotmail.com

Robson Fernando Missio

ORCID: https://orcid.org/0000-0002-8534-1175 Universidade Federal do Paraná, Brasil

E-mail: rfmissio@ufpr.br

Juliano Cordeiro

ORCID: https://orcid.org/0000-0001-8047-7463 Universidade Federal do Paraná, Brasil E-mail: julianocordeiro@ufpr.br

\begin{abstract}
Resumo
No Brasil exploração da suinocultura é uma atividade pecuária muito importante sob vários aspectos, pois, promove a geração de emprego e renda no meio rural, mas por outro lado, é responsável pela produção de grandes quantidades de dejetos como a Água Residuária da Suinocultura (ARS). Este estudo objetivou avaliar as alterações quantitativas dos principais atributos químicos do solo e nas características morfométricas de mudas de arbóreas adubadas com diferentes doses de ARS. O experimento foi conduzido na área de experimentação ambiental da Universidade Federal do Paraná - Setor Palotina. O delineamento utilizado foi em blocos casualizados (DBC) onde foram testados seis tratamentos com diferentes doses de ARS, sendo: $\mathrm{T}_{1}=0 \mathrm{~L} \mathrm{~m}^{-2}, \mathrm{~T}_{2}=1 \mathrm{~L} \mathrm{~m}^{-2}, \mathrm{~T}_{3}=2 \mathrm{~L} \mathrm{~m}^{-2}, \mathrm{~T}_{4}=3 \mathrm{~L} \mathrm{~m}^{-2}, \mathrm{~T}_{5}=4 \mathrm{~L} \mathrm{~m}^{-2}, \mathrm{~T}_{6}=5 \mathrm{~L} \mathrm{~m}^{-2}$ aplicados para adubação de mudas de Citharexylum myrianthum Cham. A aplicação do residuo resultou em aumento do percentual de matéria orgânica na camada de 0 a $20 \mathrm{~cm}$ juntamente com os teores de $\mathrm{K}^{+}, \mathrm{Mn}^{+2}, \mathrm{Zn}^{+}, \mathrm{Cu}^{+}, \mathrm{Fe}, \mathrm{CTC}$, saturação por $\mathrm{Al}^{+3}$ e $\mathrm{H}^{+3}+\mathrm{Al}^{+3}$ e diminuição do $\mathrm{pH}$, dos teores de $\mathrm{Ca}^{+2}, \mathrm{Mg}^{+2}$ e da saturação por bases. Quanto ao crescimento das mudas os maiores valores de incrementos tanto de altura como em diâmetro foram identificados nas plantas que receberam as doses de $5 \mathrm{~L} \mathrm{~m}^{-2}$ de ARS.
\end{abstract}

Palavras-chave: Adubação orgânica; Espécies arbóreas nativas; Nutrição de plantas.

\begin{abstract}
In Brazil, swine farming is a very important livestock activity in many ways, as it promotes the generation of employment and income in rural areas, but on the other hand, it is responsible for the production of large amounts of waste such as swine wastewater (SW). This study aimed to evaluate the quantitative changes in the main chemical attributes of the soil and in the morphometric characteristics of tree seedlings fertilized with different doses of swine wastewater (SW). The experiment was carried out in the environmental experimentation area of the Federal University of Paraná, Setor Palotina. The design used was in randomized blocks (RBC) where six treatments with different doses of SWW were tested, being: $\mathrm{T}_{1}=0 \mathrm{~L} \mathrm{~m}^{-2}, \mathrm{~T}_{2}=1 \mathrm{~L} \mathrm{~m}^{-2}, \mathrm{~T}_{3}=2 \mathrm{~L} \mathrm{~m}^{-2}, \mathrm{~T}_{4}=3 \mathrm{~L} \mathrm{~m}^{-2}, \mathrm{~T}_{5}=4 \mathrm{~L} \mathrm{~m}^{-2}, \mathrm{~T}_{6}=5$ $\mathrm{L} \mathrm{m}^{-2}$ applied for fertilizing Citharexylum myrianthum Cham seedlings. The application of the residue resulted in an increase in the percentage of organic matter in the layer from 0 to $20 \mathrm{~cm}$ along with the levels of $\mathrm{K}^{+}, \mathrm{Mn}^{+2}, \mathrm{Zn}^{+}, \mathrm{Cu}^{+}$, CTC, saturation by $\mathrm{Al}^{+3}$ and $\mathrm{e}^{+3}+\mathrm{Al}^{+3}$ and decrease in $\mathrm{pH}, \mathrm{Ca}^{+2}, \mathrm{Mg}^{+2}$ and base saturation levels. As for the growth of seedlings, the highest values of increments both in height and in diameter were identified in plants that received doses of $5 \mathrm{~L} \mathrm{~m}^{-2}$ of ARS.
\end{abstract}

Keywords: Organic fertilization; Native tree species; Plant nutrition.

\section{Resumen}

En Brasil, la cría de cerdos es una actividad ganadera muy importante en muchos sentidos, ya que promueve la generación de empleo y renta en las zonas rurales, pero por otro lado, es responsable de la producción de grandes cantidades de residuos como los residuos de aguas residuales porcinas (ARP). Este estudio tuvo como objetivo evaluar los cambios cuantitativos en los principales atributos químicos del suelo y en las características morfométricas de plántulas de árboles fertilizadas con diferentes dosis de aguas residuales porcinas (ARP). El experimento se realizó en el área de experimentación ambiental de la Universidad Federal de Paraná, Setor Palotina. El diseño utilizado fue en 
Bloques Aleatorizados (DBC) donde se probaron seis tratamientos con diferentes dosis de ARS, siendo: $\mathrm{T}_{1}=0 \mathrm{~L} \mathrm{~m}^{-2}$, $\mathrm{T}_{2}=1 \mathrm{~L} \mathrm{~m}^{-2}, \mathrm{~T}_{3}=2 \mathrm{~L} \mathrm{~m}^{-2}, \mathrm{~T}_{4}=3 \mathrm{~L} \mathrm{~m}^{-2}, \mathrm{~T}_{5}=4 \mathrm{~L} \mathrm{~m}^{-2}, \mathrm{~T}_{6}=5 \mathrm{~L} \mathrm{~m}^{-2}$ aplicado para la fertilización de plántulas de Citharexylum myrianthum Cham. La aplicación del residuo resultó en un aumento del porcentaje de materia orgánica en la capa de 0 a $20 \mathrm{~cm}$ junto con los contenidos de $\mathrm{K}^{+}, \mathrm{Mn}^{+2}, \mathrm{Zn}^{+}, \mathrm{Cu}^{+}, \mathrm{CTC}$, saturación por $\mathrm{Al}+3 \mathrm{y} \mathrm{H}^{+3}+\mathrm{Al}^{+3}$ y disminución de los niveles de $\mathrm{pH}, \mathrm{Ca}^{+2}, \mathrm{Mg}^{+2}$ y saturación de bases. En cuanto al crecimiento de las plántulas, los mayores valores de incrementos tanto en altura como en diámetro se identificaron en las plantas que recibieron dosis de $5 \mathrm{~L} \mathrm{~m}^{-2}$ de ARS.

Palabras clave: Fertilización orgánica; Especies de árboles nativos; Nutrición vegetal.

\section{Introdução}

A suinocultura é uma atividade da pecuária nacional que ocupa os primeiros lugares no ranking mundial de produção de animal. A expectativa é pelo aumento progressivo da produção de suínos afim de atender a demanda de consumo por esse tipo de proteína animal (USDA, 2016). Em decorrência do aumento da demanda de carne suína e de seus derivados, novas técnicas de manejos e tecnologias foram implantadas visando aumentar a produção para atender as exigências do mercado consumidor crescente. Entre tais técnicas está o confinamento, que segundo Ito et al. (2016) consiste em manter os animais em pequenas áreas a fim de potencializar a produção, mas que por outro lado, resulta na liberação de grandes volumes de dejetos.

Do ponto de vista ambiental (MAPA, 2016), pesa negativamente sob a criação de suínos as grandes quantidades de dejetos produzidos diariamente por animal cujo volume pode variar de 1,6 a 27 litros de água residuária durante as diferentes fases de manejo. Além do volume, também se deve considerar, de acordo com Ito et al. (2016), o risco de poluição devido a composição química da água residuária da suinocultura (ARS) apresentar elevados teores de fósforo e nitrogênio, metais pesados, diversas substâncias químicas como antibióticos e hormônios, podendo ainda conter diferentes tipos de micro-organismos patogênicos, entre outros.

Para Matos (2015) se a ARS for descartada de forma incorreta, pode ocasionar a contaminação dos lençóis freáticos e salinização do solo devido à grande quantidade de nitratos e sais dissolvidos, além de contaminação do solo e plantas por metais pesados. No entanto, apesar do seu potencial poluidor, após o tratamento, os dejetos podem ser utilizados como adubos orgânicos suprindo a necessidade, se não total ao menos parcial de nutrientes para as plantas, tornando o resíduo autossustentável e economicamente viável (Silva et al., 2015).

Com base na capacidade de disponibilizar nutrientes para plantas e proporcionar melhorias nas características químicas referentes à fertilidade do solo e à aplicação de ARS tem resultado no aumento na produtividade em culturais anuais, como milho (em plantio convencional e plantio direto) e soja, e no aumento na concentração de $\mathrm{Ca}^{+2}, \mathrm{~K}^{+}, \mathrm{Mg}^{+2}$ e $\mathrm{P}$ disponíveis no solo (Maggi et al., 2011; Rosa et al., 2017, Almeida et al., 2018). Para o cultivo do tomateiro Souza et al. (2010) realizaram fertirrigação com ARS e comprovaram por meio de análises foliares que todas as exigências nutricionais da cultura foram supridas. Ceretta et al. (2003) e Cabral et al. (2011) registraram aumento na concentração de $\mathrm{Ca}^{+2}, \mathrm{Mg}^{+2}$ e $\mathrm{P}$ e redução da saturação por $\mathrm{Al}^{+3}$ no perfil do solo após a aplicação de ARS em solos com pastagem natural e no cultivo com capim-elefante.

A ARS foi também aplicada para o cultivo de plantas arbóreas de interesse silvicultural como na produção de mudas de eucalipto (Pelissari et al., 2009; Batista et al., 2014), pinus (Vogel, 2002) e espécies nativas (Meira et al., 2017; Cordeiro et al., 2018; Pellá et al., 2018).

A espécie arbórea Citharexylum myrianthum Cham (Lamiaceae) é considerada heliófita apresentando bom crescimento em ambientes abertos sendo classificada como planta pioneira ou secundária inicial (IAT, 2021) o que a credencia para ser indicada para plantio em áreas de recuperação florestal (Schaffer et al., 2020). Mesmo apresentando crescimento considerado moderado à rápido se desenvolve em solo com drenagem regular e até com alagamentos sazonais, contudo, não tolera solos com elevados teor de alumínio e ácidos (Embrapa, 2021).

Considerando que a água residuária da suinocultura possui concentração química variável e que apresenta potencial para ser utilizada como adubo orgânico, este estudo objetivou avaliar as alterações quantitativas dos principais atributos químicos 
do solo e das características morfométricas (altura total e diâmetro) de mudas de Citharexylum myrianthum adubadas com diferentes doses de ARS.

\section{Material e Métodos}

O presente estudo foi implantado na Universidade Federal do Paraná (UFPR) - Setor Palotina na área de experimentação ambiental que possuiu relevo é plano com predomínio de Latossolo Vermelho de textura argilosa a muito argilosa (Embrapa, 2018). Antes da implantação do experimento a área estava ocupada por capim braquiária que foi eliminado com o uso de roçadeira mecanizada e o preparo do solo se deu através de aração com arado de discos. Em seguida foi demarcada uma área de $210 \mathrm{~m}^{2}$ que foi subdividida em seis linhas de plantio espassadas 2,50 $\mathrm{m}$ entre si. Em cada linha foram implantadas 6 blocos sendo que cada bloco foi constituído por três plantas com espaçamento de $1 \mathrm{~m}$ entre plantas e entre blocos (Figura 1).

Figura 1. Localização e disposição do plantio das mudas na área experimental.

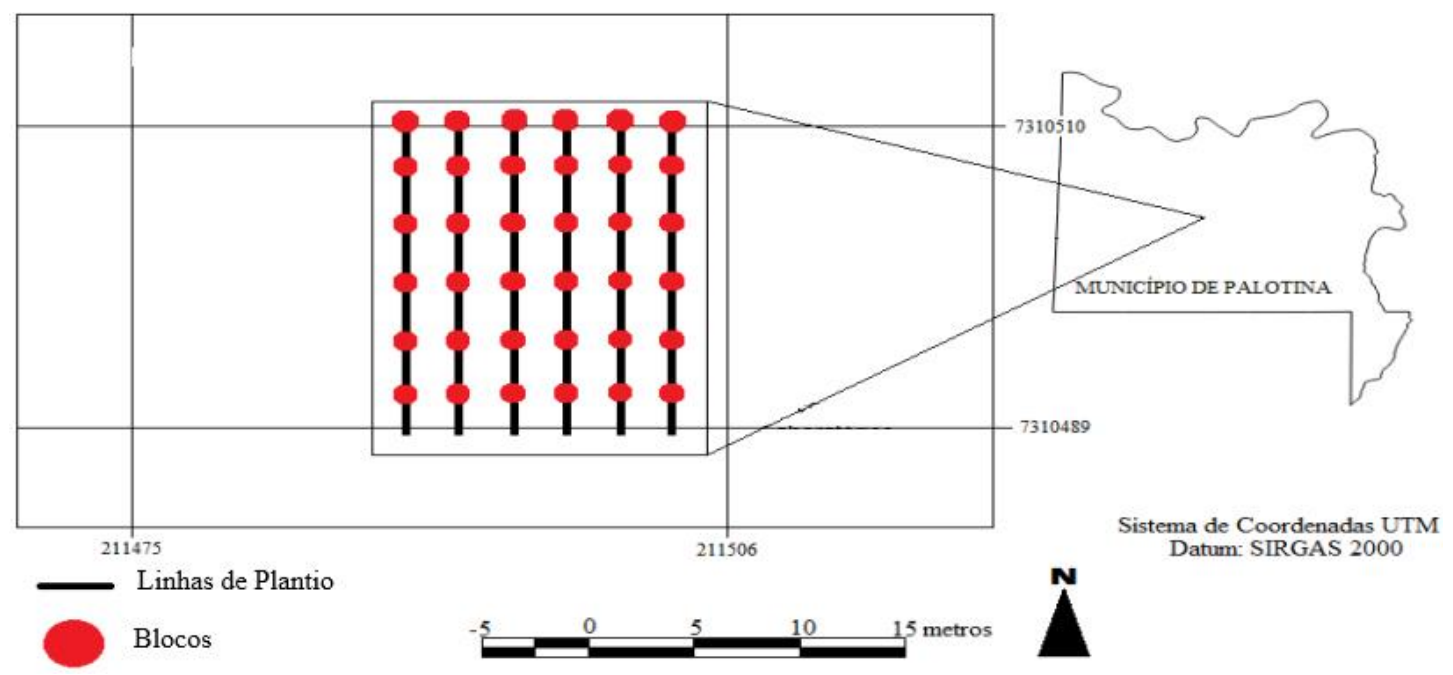

Fonte: Autores.

A escolha de Citharexylum myrianthum Cham (Lamiaceae) se deu por esta ser uma espécie de ocorrência natural em ambientes de Floresta Estacional Semidecidual do Paraná (IAT, 2021). O plantio das mudas foi realizado em covas com dimensões de $30 \times 30 \times 30 \mathrm{~cm}$ em setembro de 2017. O volume de ARS que foi aplicado em cada tratamento foi definido seguindo os estabelecidos pela legislação para licencimanento ambiental proposto por Paraná (2020). O delineamento utilizado foi em blocos casualizados, sendo que em cada bloco foi realizada a fertirrigação das mudas com diferentes doses de ARS conforme os tratamentos: Tratamento $1(\mathrm{~T} 1)=$ Controle, Tratamento 2 (T2) $=1 \mathrm{~L} \mathrm{~m}^{-2}$, Tratamento 3 (T3) $=2 \mathrm{~L} \mathrm{~m}^{-2}$, Tratamento $4(\mathrm{~T} 4)=3 \mathrm{~L} \mathrm{~m}^{-2}$, Tratamento 5 (T5) $=4 \mathrm{~L} \mathrm{~m}^{-2} \mathrm{e}$ Tratamento $6(\mathrm{~T} 6)=5 \mathrm{~L} \mathrm{~m}^{-2}$ que foram aleatoriamente distribuídos.

A mensuração das características morfométricas ocorreu aos 24 meses após o plantio seguindo critérios de Vantroba et al. (2020). A altura das plantas (m) foi mensurada com auxílio de fita métrica e o diâmetro do coleto das plantas (cm) pelo uso de paquímetro digital. Durante o período foram realizadas cinco aplicações de ARS de acordo com os tratamentos descritos anteriormente. A ARS utilizada foi adquirida em uma propriedade produtora de suínos em sistema de confinamento de matrizes e leitões. A propriedade é equipada com biodigestor e tanque de decantação de onde foi coletado o resíduo utilizado. De cada um dos três lotes de ARS aplicada foi retirada uma amostra para análise química dos teores de $\mathrm{N}, \mathrm{P}, \mathrm{K}^{+}, \mathrm{Zn}^{+}, \mathrm{Mn}^{+2} \mathrm{e} \mathrm{Cu}^{+}$que foram processadas pela metodologia da Embrapa (2009) (Tabela 1). 
Tabela 1. Concentração de macro e micronutrientes presentes na água residuária da suinocultura (ARS) utilizada na adubação das mudas arbóreas.

\begin{tabular}{|c|c|c|c|c|c|c|}
\hline \multirow{2}{*}{ Lotes de ARS } & \multicolumn{3}{|c|}{ Macronutrientes } & \multicolumn{3}{|c|}{ Micronutrientes } \\
\hline & $\mathbf{N}$ & $\mathbf{P}$ & $\mathbf{K}$ & $\mathbf{C u}$ & $\mathbf{Z n}$ & Mn \\
\hline & & & ----- & $\left.L^{-1}\right)-$ & & \\
\hline 01 & 22,40 & 4,08 & 3,81 & 0,06 & 0,26 & 0,03 \\
\hline 02 & 46,90 & 2,59 & 3,40 & 0,08 & 0,40 & 0,07 \\
\hline 03 & 39,20 & 3,72 & 3,66 & 0,05 & 0,18 & 0,05 \\
\hline
\end{tabular}

Fonte: Autores.

Para avaliar o desenvolvimento morfométrico das plantas e a eficiência dos tratamentos foi averiguada a distribuição da normalidade dos dados e dos resíduos pelo teste de Shapiro Wilk. Em seguida, os resultados das mensurações morfométricas foram submetidos à análise de variância, análise de regressão e teste Tukey $(\mathrm{P}<0,05)$. Todos os testes foram executados pelos programas PAST4.03 ${ }^{\circledR}$ (Hammer e Ryan, 2001) e SISVAR ${ }^{\circledR}$ (Ferreira, 2014).

\section{Resultados e Discussão}

\subsection{Características químicas do solo}

Comparativamente, ocorreu diminuição dos valores dos atributos $\mathrm{pH}$, das bases trocáveis $\left(\mathrm{Ca}^{+2}\right.$ e $\left.\mathrm{Mg}^{+2}\right)$ e da saturação por bases (V\%) de T1 em relação aos demais tratamentos (Tabela 2). Por outro lado, teve-se o aumento dos valores de $\mathrm{Al}^{+3}$, $\mathrm{H}^{+}+\mathrm{Al}^{+3}, \mathrm{~K}^{+}, \mathrm{C}, \mathrm{P}$ e dos micronutrientes $\left(\mathrm{Cu}^{+}, \mathrm{Mn}^{+2}, \mathrm{Zn}^{+}\right.$e Fe$\left.{ }^{+3}\right)$ e da CTC do solo com o aumento das doses de ARS (Tabela 2).

Tabela 2. Atributos químicos de fertilidade do solo obtidos mediante a adubação com diferentes doses de ARS.

\begin{tabular}{|c|c|c|c|c|c|c|c|c|c|}
\hline \multirow[b]{2}{*}{ Tratamentos } & pH & $\mathbf{A l}^{+3}$ & $\mathbf{H}^{+}+\mathbf{A l}^{+3}$ & $\mathbf{C a}^{+2}$ & $\mathrm{Mg}^{+2}$ & $\mathbf{K}^{+}$ & CTC & $\mathbf{V}$ & $\mathbf{m}$ \\
\hline & \multicolumn{9}{|c|}{ 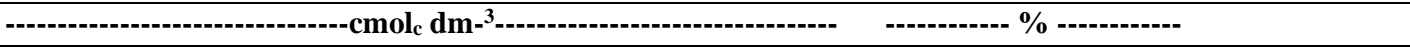 } \\
\hline T1 & 4,86 & 0,10 & 4,53 & 4,87 & 1,98 & 0,47 & 11,84 & 61,73 & 1,35 \\
\hline $\mathrm{T} 2$ & 4,69 & 0,10 & 6,69 & 4,42 & 1,93 & 0,77 & 13,81 & 51,57 & 1,38 \\
\hline $\mathrm{T} 3$ & 4,62 & 0,10 & 5,10 & 4,62 & 1,93 & 0,70 & 12,35 & 58,69 & 1,36 \\
\hline $\mathrm{T} 4$ & 4,49 & 0,20 & 5,18 & 3,87 & 1,56 & 0,74 & 11,35 & 54,37 & 3,14 \\
\hline T5 & 4,49 & 0,20 & 7,76 & 4,07 & 1,60 & 0,90 & 14,41 & 46,15 & 2,92 \\
\hline \multirow[t]{3}{*}{ T6 } & 4,68 & 0,15 & 7,14 & 4,37 & 1,70 & 0,84 & 13,95 & 48,81 & 2,16 \\
\hline & $\mathbf{C}$ &  & & & Mn & & & & \\
\hline & $\mathrm{g} \mathrm{dm}^{-3}$ & \multicolumn{8}{|c|}{ 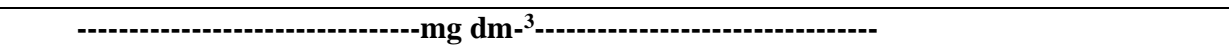 } \\
\hline $\mathrm{T} 1$ & 23,24 & \multicolumn{2}{|c|}{5,25} & 9,80 & 155,0 & \multicolumn{2}{|c|}{14,5} & \multicolumn{2}{|c|}{30,7} \\
\hline $\mathrm{T} 2$ & 23,24 & \multicolumn{2}{|c|}{12,22} & & 180,0 & \multicolumn{2}{|c|}{15,0} & \multicolumn{2}{|c|}{31,7} \\
\hline $\mathrm{T} 3$ & 24,61 & \multicolumn{2}{|c|}{9,87} & & 188,0 & \multicolumn{2}{|c|}{13,2} & \multicolumn{2}{|c|}{35,1} \\
\hline $\mathrm{T} 4$ & 24,61 & \multicolumn{2}{|c|}{14,40} & & 212,0 & \multicolumn{2}{|c|}{11,5} & \multicolumn{2}{|c|}{40,0} \\
\hline T5 & 24,61 & \multicolumn{2}{|c|}{19,80} & & 178,0 & \multicolumn{2}{|c|}{11,9} & \multicolumn{2}{|c|}{42,5} \\
\hline T6 & 27,34 & \multicolumn{2}{|c|}{20,67} & & 164,0 & \multicolumn{2}{|c|}{19,0} & \multicolumn{2}{|c|}{34,1} \\
\hline
\end{tabular}

SB: Soma de bases; CTC: Capacidade de troca catiônica; V\%: Saturação por Bases. T1 = Controle; T2: 1,0 L m²; T3: 2,0 L m²; T4: 3,0 L m 2; T5: 4,0 L m $\mathrm{L} \mathrm{e} \mathrm{T6:} \mathrm{5,0} \mathrm{L} \mathrm{m}^{-2}$. Fonte: Autores.

De acordo com a composição química da ARS (Tabela 1), a sua aplicação pode acrescentar ao solo quantidades variáveis de macro e micronutrientes. Para Gatiboni e Nicolosso (2019) a variação da concentração dos nutrientes na ARS é muito comum, pois, está relacionada com a origem do dejeto que varia de acordo com o sistema de produção adotado, categoria animal, o tipo de manejo dos animais e do dejeto, do volume de água utilizada por animal/dia, entre outros fatores. Sobre os micronutrientes, 
o $\mathrm{Zn}^{+}$foi o que apresentou maior concentração na ARS comparativamente ao $\mathrm{Mn}^{+2} \mathrm{e} \mathrm{Cu}^{+}$(Tabela 1). A presença desses elementos nos dejetos de suínos é oriunda, segundo Barros et al. (2019), da suplementação mineral adicionada às rações e também podem ser encontrados na formulação de medicamentos administrados durante o manejo dos suínos.

Quanto as alterações químicas do solo em decorrência da aplicação crescente de ARS tem-se o aumento da MO do solo que, segundo Leite (2004), depende da quantidade de compostos orgânicos presentes no resíduo o que pode explicar a ausência ou a pequena variação desse percentual para algumas das doses testadas. Outra explicação está ligada à sedimentação dos sólidos presentes no resíduo durante o armazenamento ocasionando redução dos valores da MO do solo conforme também comprovado por Scherer et al. (2010).

O solo que recebeu a dose de $3 \mathrm{~L} \mathrm{~m}^{-2}$ apresentou menor valor de CTC com $11,35 \mathrm{cmol}_{\mathrm{c}} \mathrm{dm}^{-3}$, enquanto a aplicação de 4 $\mathrm{L} \mathrm{m}^{-2}$ registrou o maior valor $\left(14,41 \mathrm{cmol}_{\mathrm{c}} \mathrm{dm}^{-3}\right.$ ) (Tabela 2). A capacidade de troca catiônica corresponde a quantidade de cargas negativas proporcionadas pela presença de coloides formados por pequenas partículas de argila e matéria orgânica que atuam na retenção e disponibilização de cátions para a solução do solo. De acordo com Prezotti e Martins (2013) a manutenção e/ou aumento da CTC do solo atua positivamente na estabilidade dos agregados do solo, bem como, contribui para elevação do $\mathrm{pH} e$ maior disponibilização de nutrientes para as plantas.

Para saturação por bases (V\%) o maior percentual foi observado no solo do tratamento controle (V\% $=61,73 \%)$ decaindo em função do aumento das doses, chegando a $\mathrm{V} \%=46,15 \%$ no solo que recebeu a dose de $4 \mathrm{~L} \mathrm{~m}^{-2}$ (Tabela 2). Comparativamente, como foi observado por Caovilla et al. (2010) estes não constataram alterações significativas em solo cultivado com soja para os teores de V\% após aplicação de diferentes doses de ARS. O percentual de saturação por alumínio $(\mathrm{m} \%)$ apresentou maior valor no solo que recebeu o tratamento T4 $(\mathrm{m} \%=3,14 \%)$ e o menor observado no T1 $(\mathrm{m} \%=1,35 \%)$. Houve um aumento da saturação por $\mathrm{Al}^{+3}$ em função do aumento das doses em contraposição do que ocorreu com a saturação por bases, processo este também identificado por Homem et al. (2014). De acordo com Ronquim (2010) baixos teores de saturação por bases são ocasionados pela pequena quantidade de cargas negativas do solo que interagem com cátions básicos, tais como: $\mathrm{Ca}^{+2}, \mathrm{Mg}^{+2}, \mathrm{~K}^{+} \mathrm{e} \mathrm{Na}^{+}$ao passo que as demais cargas interagem com cátions ácidos como $\mathrm{Al}^{+3}$, o que tende a elevar o $\mathrm{m} \%$. Tal princípio explica a diferença para os valores de $\mathrm{V} \%$ e $\mathrm{m} \%$, uma vez que a diminuição de cátions básicos interagindo com cargas negativas proporcionaram maior interação das cargas negativas com o cátion $\mathrm{Al}^{+3} \mathrm{o}$ que elevou o $\mathrm{m} \%$.

$\mathrm{O}$ pH do solo variou de 4,87 (Controle) à 4,49 para os tratamentos T4 e T5 (Tabela 2). O comportamento decrescente do pH do solo em função do aumento das doses de ARS com consequente aumento de acidez do solo pode ser segundo Caires (2013) decorrente da decomposição da matéria orgânica. A decomposição pelos microrganismos do solo libera ácidos (orgânicos e carbônico) que elevam a acidez. $\mathrm{O}$ aumento da acidez resulta na elevação dos teores de cátions ácidos $\left(\mathrm{Al}^{3+}\right.$ e íons $\mathrm{H}^{+}$) que passam a interagir com as cargas negativas dos coloides provocando a diminuição das interações entre os cátions básicos que por sua vez reduzem a saturação por bases e consequentemente a disponibilidade de nutrientes para as plantas. Ceretta et al. (2003) em trabalhos realizados com adubação utilizando ARS observaram que os valores de $\mathrm{pH}$ do solo pouco se alteraram e

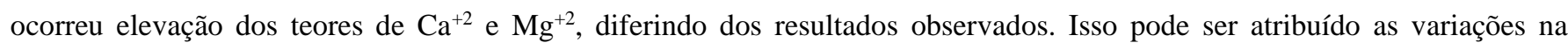
composição dos resíduos da atividade suinícola, sendo este dependente do manejo adotado. Esses autores não constataram variações significativas nos valores de $\mathrm{pH}$ em profundidade de 0 a $20 \mathrm{~cm}$ após a aplicação de ARS para adubação de soja.

Quanto ao $\mathrm{Ca}^{+2}$ no solo este variou entre os tratamentos desde 4,87 $\mathrm{cmol}_{\mathrm{c}} \mathrm{dm}^{-3}$ (Controle) até $3,87 \mathrm{cmol}_{\mathrm{c}} \mathrm{dm}^{-3}$ (solo do T4). De certa forma, a redução dos teores de $\mathrm{Ca}^{+2}$ ocorreu com o aumento das doses de ARS (Tabela 2). De acordo com Prezotti \& Martins (2013) essa diminuição está associada ao aumento da acidez, devido ao baixo teor de cátions básicos e prevalência de cátions ácidos como $\mathrm{Al}^{3+}$. Quanto ao $\mathrm{Mg}^{+2}$ o maior teor foi observado em $\mathrm{T} 1\left(1,98 \mathrm{cmol}_{\mathrm{c}} \mathrm{dm}^{-3}\right)$ e o menor valor observado no solo de T4 $\left(1,56 \mathrm{cmol}_{\mathrm{c}} \mathrm{dm}^{-3}\right)$. A diminuição dos teores deste nutriente em função das doses, seguiu o mesmo comportamento do $\mathrm{Ca}^{+2}$, uma vez que ambos os elementos estão relacionados com a acidez do solo como exposto anteriormente. 
Para o $\mathrm{K}^{+}$a sua concentração no solo foi diretamente proporcional ao aumento das dosagens de ARS (Tabela 2), uma vez que no solo do T5 foi registrada a maior concentração deste elemento $\left(0,90 \mathrm{cmol}_{\mathrm{c}} \mathrm{dm}^{-3}\right)$ e em T1 a menor $\left(0,47 \mathrm{cmol}_{\mathrm{c}} \mathrm{dm}^{-3}\right)$. $\mathrm{O}$ aumento do teor de $\mathrm{K}^{+}$no solo em função das doses apresenta um comportamento distinto quando comparado aos nutrientes $\mathrm{Ca}^{+2} \mathrm{e} \mathrm{Mg}^{+2}$. O aumento ocorreu pela presença de $\mathrm{K}^{+}$solúvel no resíduo utilizado que segundo Diesel et al. (2002) é porque a ARS apresenta em sua composição minerais dissolvidos, entre eles o $\mathrm{K}^{+}, \mathrm{Ca}^{+2} \mathrm{e} \mathrm{Mg}^{+2}$. Erthal et al. (2010) evidenciaram o aumento nas concentrações de $\mathrm{Ca}^{+2}, \mathrm{Mg}^{+2}$ e K${ }^{+}$em argissolo após fertirrigação com ARS. O aumento do teor de $\mathrm{K}^{+}$e diminuição do teor de $\mathrm{Mg}^{+2}$ após sucessivas aplicações de esterco líquido de suínos em solo cultivado com gramineas forrageiras também foi constatado por Queiroz et al. (2004).

Os teores de $\mathrm{Cu}^{+} \mathrm{e} \mathrm{Zn}^{+}$no solo foram classificados como alto (Prezotti \& Martins, 2013) em todos os blocos, inclusive para o controle (Tabela 2), o que indica a presença desses elementos em condições naturais do solo (sem tratamento). Os teores de $\mathrm{Cu}^{+}$variaram entre 9,80 e 12,60 $\mathrm{mg} \mathrm{dm}^{-3}$ (Tabela 2). Tais valores não indicam que essas concentrações possam ocasionar algum risco ambiental (Paraná, 2020). Considera-se que o $\mathrm{Cu}^{+}$se encontra em forma solúvel quando presente em resíduos suinícolas, no entanto, os processos químicos como a decomposição da matéria orgânica podem influenciar na dinâmica deste elemento influenciando em sua concentração no solo (Dhaliwal et al., 2019).

Para o micronutriente $\mathrm{Zn}^{+}$o maior valor foi observado no solo que recebeu a dose de $5 \mathrm{~L} \mathrm{~m}^{-2}\left(19,0 \mathrm{mg} \mathrm{dm}^{-3}\right)$ coincidindo com a dose que proporcionou maior incremento de $\mathrm{Cu}^{+}$(Tabela 2). O menor valor foi observado no solo que recebeu a dose de $3 \mathrm{~L} \mathrm{~m}^{-2}\left(14,5 \mathrm{mg} \mathrm{dm}^{-3}\right)$. Smanhotto et al. (2010) observaram aumento do teor de $\mathrm{Zn}^{+}$no solo após aplicações sucessivas de ARS, ou seja, as maiores doses também contribuíram para o aumento desse elemento no solo. Os teores de $\mathrm{Mn}^{+2}$ apresentam classificação alta para todas as doses testadas, incluindo a testemunha. Entre os micronutrientes $\mathrm{o} \mathrm{Mn}^{+2}$ foi o que apresentou maior concentração no solo (Tabela 2), possivelmente devido à grande concentração deste elemento no resíduo utilizado para a adubação. O maior teor foi registrado no solo que recebeu o T4 $\left(212,00 \mathrm{mg} \mathrm{dm}^{-3}\right)$ e o menor valor foi em T1 (155,00 $\left.\mathrm{mg} \mathrm{dm}^{-3}\right)$. Silva et al., (2016) constataram a diminuição do teor de $\mathrm{Cu}^{+}, \mathrm{Mn}^{+2}$ e $\mathrm{Zn}^{+}$em solo tratado da água residuária da bovinocultura e apontaram que a presença de matéria orgânica indisponibiliza tais elementos devido o processo de quelação provocando a redução de íons desses elementos livres no solo.

Segundo Miyazawa e Barbosa (2015) como a ARS possui muitos nutrientes necessários para o desenvolvimento das plantas isso a qualifica para ser empregada como adubo, pois, tais nutrientes após absorvidos e metabolizados são empregados para a construções de suas biomoléculas resultando no incremento de suas variáveis morfométricas. Contudo, a aplicação de ARS como adubação deve considerar a legislação ambiental que estabelece os valores máximos permitidos para concentração de metais pesados no solo (Paraná, 2020). Os teores de $\mathrm{Cu}^{+}, \mathrm{Zn}^{+}$, entre outros, presentes na ARS devem ficar abaixo dos valores de referência, uma vez que esses procedimentos visam reduzir os riscos de contaminação dos solos, dos corpos d'água superficiais e dos lençóis freáticos e aquíferos (Rosa et al. 2017).

\subsection{Uso da ARS e desenvolvimento das mudas}

Com relação as variáveis morfométricas os resultados da Tabela 3 mostraram existência de diferença estatística para a altura $\left(\mathrm{F}_{(0,05 ; 5,25)}=3,392\right)$ e para o diâmetro $\left(\mathrm{F}_{(0,05 ; 5,25)}=8,123\right)$ das mudas de Citharexylum myrianthum. 
Tabela 3. Resultados da análise de variância, regressão e teste Tukey para as variáveis morfométricas de altura e diâmetro.

\begin{tabular}{|c|c|c|c|c|c|c|c|}
\hline ANOVA & $\mathbf{F}$ & $\mathbf{P}$ & Regressão & Equação & $\mathbf{R}^{2}$ & \multicolumn{2}{|c|}{ CV\% } \\
\hline Altura (m) & 3,392 & $0,0178^{*}$ & $\mathrm{~L}$ & $y=0,1095 x+2,3046$ & 0,3461 & \multicolumn{2}{|c|}{17,07} \\
\hline Diâmetro (cm) & 8,123 & $0,0001 * *$ & $\mathrm{~L}$ & $y=0,4198 x+5,1007$ & 0,8637 & \multicolumn{2}{|c|}{11,85} \\
\hline TUKEY & & \multicolumn{6}{|c|}{ Tratamentos } \\
\hline & & T1 & $\mathbf{T}$ & T3 & T4 & T5 & T6 \\
\hline Altura (m) & & $2,28 a b$ & & $2,02 \mathrm{~b}$ & $2,67 a b$ & $2,76 a b$ & $2,93 \mathrm{a}$ \\
\hline Diâmetro (cm) & & $4,70 \mathrm{~b}$ & & $5,94 \mathrm{ab}$ & $6,76 \mathrm{a}$ & $6,74 \mathrm{a}$ & $6,91 \mathrm{a}$ \\
\hline
\end{tabular}

m: metro; cm: diâmetro; L: comportamento linear. $\mathrm{R}^{2}$ : Coeficiente de determinação do modelo; CV\% - coeficiente de variação; $*=$ significativo a $5 \%$ de probabilidade $* *=$ significativo a $1 \%$ de probabilidade. Médias seguidas de letras iguais nas linhas não diferem estatisticamente pelo teste de Tukey a 5\% de probabilidade. Fonte: Autores.

A comparação entre as médias das variáveis analisadas mostrou que o uso da ARS em doses crescentes contribuiu para o incremento tanto de altura como de diâmetro das mudas (Tabela 3). Foi possível verificar que para a altura as mudas responderam ao tratamento 6 com maior crescimento $(\mathrm{T} 6=2,93 \mathrm{~m})$, já para o diâmetro a aplicação de doses a partir de 3,0 L m ${ }^{2}$ até $5,0 \mathrm{~L} \mathrm{~m}^{-2}(\mathrm{~T} 4=6,64 \mathrm{~cm}, \mathrm{~T} 4=6,76 \mathrm{~cm}$ e T6=6,91 cm) proporcionaram às mudas os melhores incrementos. As equações de regressão (Figura 2) para as variáveis altura e diâmetro apresentaram comportamento linear, sendo significativas a 5\% de probabilidade. Os coeficientes de determinação $\left(\mathrm{R}^{2}\right)$ explicaram 34,6\% da variação da altura das mudas e 86,3\% do diâmetro.

A aplicação de ARS nas mudas de Citharexylum myrianthum promoveu efeitos nutricionais positivos resultando em maior crescimento tanto em altura como em diâmetro. Contudo, o uso da ARS como via de adubação orgânica para esta espécie não tem sido explorado nos estudos conduzidos a campo que se dedicaram em avaliar o crescimento das mudas plantadas em diferentes espaçamento e tipos de solos (Torres et al. 2009), resistência aos rigores climáticos como geadas (Vieira et al. 2009), condições de relevo e disponibilidade hídrica (Silveira et al. (2013) e influência do tamanho dos recipientes de produção de mudas (Morais Júnior et al., 2020).

Figura 2. Variação na altura e diâmetro médio das mudas de Citharexylum myrianthum em função da fertirrigação com água residuária de suinocultura (ARS).

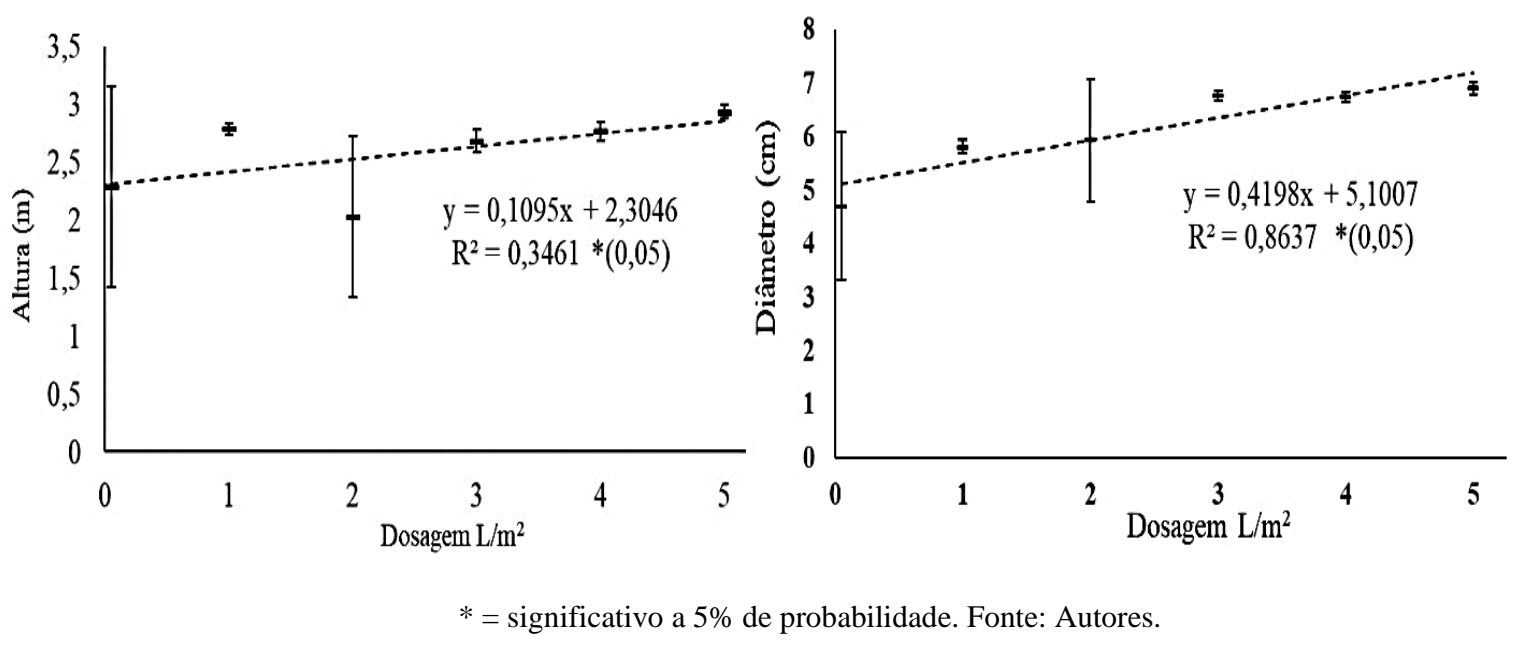

No caso de Citharexylum myrianthum o uso da ARS resultou em maiores crescimento em razão do aumento das doses até o limite testado de $5 \mathrm{~L} \mathrm{~m}^{-2}$. Para a variável altura, o incremento médio foi $0,65 \mathrm{~m}$ enquanto para o diâmetro chegou $2,21 \mathrm{~cm}$ comparativamente entre os tratamentos T6 e T1. Os efeitos de ARS sobre o crescimento de mudas arbóreas também foram encontrados por Batista et al. (2014) para mudas de eucalipto e Pelissari et al. (2009) que além do maior incremento em altura e diâmetro citaram que a ARS possibilitou antecipar o tempo de produção das mudas de eucalipto em 30 dias. Souza et al. (2006) 
por sua vez identificaram resultados semelhantes para mudas arbóreas de Cedrela odorata L. e Schinus terebinthifolius Raddi pelo uso de diferentes tipos de adubos orgânicos. Esses autores destacaram que o plantio de espécies nativas para fins comerciais necessita ainda de estudos sobre o comportamento silvicultural das espécies, principalmente, no que tange suas necessidades nutricionais, aspecto esse que pode ser extrapolado para às plantas que se destinam à recuperação de áreas de reserva legal e/ou preservação permanente. Para o mogno africano Araújo et al. (2019) constataram que a aplicação de ARS em concentração de até $50 \%$ produziram melhores resultados tanto no crescimento como no equilíbrio nutricional entre as diferentes partes da biomassa das mudas cultivadas.

De modo geral, as necessidades nutricionais de Citharexylum myrianthum ainda requerem pesquisa (Sorreano et al. 2012) para que a recomendação de adubação mineral ou orgânica venha suprir ou complementar essas necessidades e evitar deficiências nutricionais. Para Brun et al. (2017) no caso das espécies arbóreas as exigências quanto a determinados nutrientes é um dos fatores que podem explicar as variações no seu crescimento, existindo lacunas ligadas aos limites nutricionais principalmente com relação aos micronutrientes. Contudo, existem outros condicionantes ao crescimento das plantas como aqueles relacionados às características intrínsecas, como o patrimônio genético das sementes que podem ser diferentes em decorrência processo de fecundação cruzada que ocorre mesmo nas sementes produzidas por uma mesma árvore matriz (Carvalho, 2008).

\section{Conclusão}

A aplicação da ARS alterou quantitativamente os atributos químicos do solo com o aumento do percentual de matéria orgânica, capacidade de troca catiônica, elevação da saturação por $\mathrm{Al}^{+3}$, da acidez e do teor de $\mathrm{K}^{+}$na camada de 0 a $20 \mathrm{~cm}$. Também, provocou a diminuição dos teores de $\mathrm{Ca}^{+2}$ e $\mathrm{Mg}^{+2}$ e da saturação por bases. Quanto a presença de metais no solo a adubação utilizando ARS mostrou aumento dos teores de $\mathrm{Cu}^{+}, \mathrm{Zn}^{+}$e $\mathrm{Mn}^{+2}$.

Para as mudas arbóreas os maiores incrementos de altura e diâmetro foram identificados nas plantas que receberam $5 \mathrm{~L}$ $\mathrm{m}^{-2}$ de ARS. A utilização de ARS via de adubação orgânica deve ser precedida pela observância das recomendações preconizadas pela legislação ambiental vigente para se evitar alterações negativas na fertilidade do solo e contaminação dos solos e águas.

\section{Referências}

Almeida, A. C. S., Santos, H. H. O., Bortolo, D. P., Lourente, E. R. P., Cortez, J. W., \& Oliveira, F. C. (2018). Soil physical properties and yield of soybean and corn grown with wastewater. Revista Brasileira de Engenharia Agricola e Ambiental, 22: 843-848. http://dx.doi.org/10.1590/1807-1929/agriambi.v22n12p843848

Araújo, E. F., Arauco, M. A. S., Dias, B. A. S, Lacerda, J. J. J., Boechat, C. L., Porto, D. L., \& Arauco, L.R. R. (2019). Wastewater from swine farming in the growth and nutrition of Khaya senegalensis (Desr.) A Juss seedlings. Bioscience Journal, 35 (5), 1378-1389. https://doi.org/10.14393/BJ-v35n5a2019-42276

Barros, F. M., Martinez, M. A., Neves, J. C. L, Matos, A. T., \& Silva, D. D. (2005). Características químicas do solo influenciadas pela adição de água residuária da suinocultura. Revista Brasileira de Engenharia Agrícola $e^{2}$ Ambiental, <http://www.agriambi.com.br/revista/suplemento/index_arquivos/PDF/047.pdf?script=sci_pdf\%C0\%03d=S1415-

$43662005000400004 \& \operatorname{lng}=\mathrm{en} \& \mathrm{nrm}=\mathrm{iso} \& \operatorname{lng}=\mathrm{pt}>$. Acesso em 20 abr. 2021.

Batista, R., Martinez, M., Paiva, H., Batista, R., \& Cecon, P. (2014). Efeito da água residuária da suinocultura no desenvolvimento e qualidade de mudas de Eucalyptus urophylla. Ciência Florestal, 24(1), 127-135. doi:https://doi.org/10.5902/1980509813330

Brun, F. G. K., Brun E. L., Gerber, J., Szymczak, D. A., Londero, E. K.; Meyer, E. A., \& Navroski, M. C. (2017). Nutrition facts and limits for micronutrients in tree species used in urban forestry. Anais da Academia Brasileira de Ciências, 89, 1881-1893. https://doi.org/10.1590/0001-3765201720160785

Cabral, J. R., Freitas, P. S. L., Rezende, R., Muniz, A. S., \& Bertonha, A. (2011). Impacto da água residuária de suinocultura no solo e na produção de capimelefante. Revista Brasileira de Engenharia Agrícola e Ambiental, 15(8), 823-831. https://doi.org/10.1590/S1415-43662011000800009

Caires, E. F. (2013). Correção da Acidez do Solo em Sistema Plantio Direto. Informações Agronômicas, 141: 1-13.

Caovilla, F. A., Sampaio, SC., Smanhotto, A., Nóbrega, L. H. P., Queiroz, M. M. F., \& Gomes, B. M. (2010). Características químicas de solo cultivado com soja e irrigado com água residuária da suinocultura. Revista Brasileira de Engenharia Agrícola e Ambiental, 14, 692-697. https://doi.org/10.1590/S141543662010000700002 
Ceretta, C. A., Durigon, R., Basso, C. J., Barcellos, L. A. R., \& Vieira, F. C. B. (2003). Características químicas de solo sob aplicação de esterco líquido de suínos em pastagem natural. Pesquisa agropecuária brasileira, 38(6), 729-735. https://doi.org/10.1590/S0100-204X2003000600009

Carvalho, P. E. R. (2008) Açoita-Cavalo (Luehea divaricata). Colombo: Embrapa Florestas, (Embrapa Florestas. Circular técnica, 147). 9 p.

Cordeiro, J., Sanchez, L. R., Santos, W. R. P., Missio, R. F., \& Pavlak, R. J. (2018). Growth of forest seedlings submitted to fertilization with swine wastewater. Brazilian Journal of Development, 4, 3862-3875.

Dhaliwal, S. S., Naresh, R. K., Mandal, A., Singh, R., \& Dhaliwal, M. K. (2019). Dynamics and transformations of micronutrients in agricultural soils as influenced by organic matter build-up: A review. Environmental and Sustainability Indicators, 1-2. https://doi.org/10.1016/j.indic.2019.100007

Diesel, R. Miranda, C. R., \& Perdomo, C. C. (2002). Coletânea de tecnologias sobre dejetos suínos. Concórdia: Embrapa Suínos e Aves, 30 p.

Empresa Brasileira de Pesquisa Agropecuária - Embrapa (2009). Manual de análises químicas de solos, plantas e fertilizantes. 2. ed. Brasília, DF: Embrapa Informação Tecnológica, $627 \mathrm{p}$.

Empresa Brasileira de Pesquisa Agropecuária - Embrapa. (2018). Sistema Brasileiro de Classificação de Solos - SBCS. (2018). 5. Ed. Brasília, DF: Embrapa Solos. 356 p.

Empresa Brasileira de Pesquisa Agropecuária - Embrapa (2021). Árvores na agricultura. Embrapa-Agrobiologia. <https://www.embrapa.br/agrobiologia/arvores-na-agricultura/especies>. Acesso em: 26 abr. 2021.

Erthal, V. J. T., Ferreira, P. A., Matos, A.T., \& Pereira, O. G. (2010). Alterações físicas e químicas de um Argissolo pela aplicação de água residuária de bovinocultura. Revista Brasileira de Engenharia Agrícola e Ambiental, 14(5): 467-477.

Ferreira, D. F. (2014). SISVAR: a Guide for its Bootstrap procedures in multiple comparisons. Ciência Agrotécnica, 38(2), 109-112.

Gatiboni, L. C., \& Nicoloso, R. S. (2019). Uso de dejetos animais como fertilizante: impactos ambientais e a experiência de Santa Catarina. In: Palhares, JCP. (Ed) Produção animal e recursos hídricos: tecnologias para manejo de resíduos e uso eficiente dos insumos. Brasília, DF: Embrapa. p. $79-98$.

Hammer, Ø. H., Harper, D. A. T., \& Ryan, P. D. (2001). PAST: Paleontological statistics software package for education and data analysis. Palaeontologia Electronica, 4(1), 1-9. < http://palaeo-electronica.org/2001_1/past/issue1_01.htm. Acesso em: 10 jan. 2021.

Homen, B. G. C., Almeida Neto, O. B., Condé, M. S., Silva, M. D., \& Ferreira, I. M. (2014). Efeito do uso prolongado de água residuária da suinocultura sobre as propriedades químicas e físicas de um Latossolo Vermelho-Amarelo. Cientifica, 42(3), 299-309. < http://www.cientifica.org.br/index.php/cientifica/article/viewFile/507/349>. Acesso em: 24 fev. 2021.

Instituto Água e Terra - IAT (2021). Espécies Produzidas nos Viveiros. 2021. <http://www.iat.pr.gov.br/Pagina/Especies-Produzidas-nos-Viveiros\#>. Acesso em: 24 fev. 2021

Ito, M., Guimarães, D. D., \& Amaral, G. F. (2016) Impactos ambientais da suinocultura: desafios e oportunidades. BNDES Setorial, $44: 125156$.

Leite, L. F. C. (2004). Matéria orgânica do solo. Embrapa Meio-Norte. 31 p. <https://ainfo.cnptia.embrapa.br/digital/bitstream/item/36210/1/Doc97.pdf>. Acesso em: 15 jun. 2021.

Maggi, C. F., Freitas, P. S. L., Sampaio, S. C., \& Dieter, J. (2011). Lixiviação de nutrientes em solo cultivado com aplicação de água residuária de suinocultura. Revista Brasileira de Engenharia Agrícola e Ambiental, 15(2), 170-177, 2011. https://doi.org/10.1590/S1415-43662011000200010

Ministério da Agricultura, Pecuária e Abastecimento - MAPA (2016). Suinocultura de Baixo Carbono - Tecnologias de produção mais limpa e aproveitamento econômico dos resíduos da produção de suínos. Brasília: MAPA. 96 p.

Matos, A. T., Sediyama, M. A. N., Freitas, S. P., Vidigal., S. M., \& Garcia, N. C. P. (2015). Características químicas e microbiológicas do solo influenciadas pela aplicação de dejeto líquido de suínos. Ceres, 44(254). < http://www.ceres.ufv.br/ojs/index.php/ceres/article/view/2432/434>. Acesso em: 24 jul. 2021.

Meira, D., Rubin, H. M., Bordin, R., Dagios, R. F., Meier, C., Aguir, A. C. M., \& Cantarelli, E. B. (2017). Avaliação de diferentes substratos no desenvolvimento inicial de viveiro do angico vermelho (Anadenanthera macrocarpa). Revista Cultivando o Saber, 10, 90-96.

Miyazawa, M., \& Barbosa, G. M. C. (2015). Dejeto líquido de suíno como fertilizante orgânico: método simplificado. Boletim Técnico. n. 84 . IAPAR. 26 p.

Morais Junior, V. T. M. M., Jacovine, L. A. G., Oliveira, K., Albuquerque, T. P, Faustino, I. S., Silva, L. B., Alves, E. B. B. M, Torres, C. M. M. E, de Paiva, H. N., Cruz, R. A. (2020). Performance of Five Native Atlantic Forest Species Planted in Containers of Different Size for Restoring Degraded Areas in Minas Gerais. Forests, 11(937). https://doi.org/10.3390/f11090937

Paraná. (2020). Resolução SEDEST $n^{\circ} 15$, de 5 de março de 2020. Estabelece condições e critérios e adota outras providencias, para o licenciamento ambiental de Empreendimentos de Suinocultura no Estado do Paraná. Curitiba. 2020.

Pelissari, R. A. Z., Sampaio, S. C., Gomes, S. D., \& Crepalli, M. S. (2009). Lodo têxtil e água residuária da suinocultura na produção de mudas de Eucalyptus grandis (W, Hill ex Maiden). Engenharia Agrícola, 29(2), 288-300. https://doi.org/10.1590/S0100-69162009000200012

Pellá, M., Araújo, G. V., \& Cordeiro, J. (2018). Morphometric Increase of Tree Species Submitted to Fertilization With Swine Wastewater. Scientia Agraria Paranaensis, 17, 368-373.

Prezotti, L. C., \& Martins, A. G. (2013). Guia de interpretação de análise de solo e foliar. Incaper. 104 p.

Queiroz, F. M., Matos, A. T., Pereira, O. G., \& Oliveira, R. A. (2004). Características químicas de solo submetido ao tratamento com esterco líquido de suínos e cultivado com gramíneas forrageiras. Ciência Rural, 34(5), 1487-1492. 〈https://scielo.br/pdf/cr/v34n5/a24v34n5.pdf>. 
Research, Society and Development, v. 11, n. 2, e53411223043, 2022

(CC BY 4.0) | ISSN 2525-3409 | DOI: http://dx.doi.org/10.33448/rsd-v11i2.23043

Ronquim, C. C. (2010). Conceitos de fertilidade do solo e manejo adequado para as regiões tropicais. Embrapa Monitoramento por Satélite. Boletim de Pesquisa e Desenvolvimento, $26 \mathrm{p}$.

Rosa, D. M., Sampaio, S. C., Pereira, P. A. M., Mauli, M. M., \& Reis, R. R. (2017). Swine Wastewater: Impacts on Soil, Plant, and Leachate. Engenharia Agrícola, 37(5), 928-939. https://doi.org/10.1590/1809-4430-eng.agric.v37n5p928-939/2017

Rosa, D. M., Sampaio, S. C., Pereira, P. A. M., Reis, R. R. dos., \& Sbizzaro, M. (2017). Corn fertilization using swine wastewater and soil-water environmental quality. Eng. Agríc., Jaboticabal, 37(4): 801-810. http://dx.doi.org/10.1590/1809-4430-Eng.Agric.v37n4p801-810/2017

Schäffer, L. H., Mattar, E. A., Nakajima, N. Y., Silva, A. S., Borges, R. A., Borges, A. V. P., Carpanezzi, A. A., Neves, E. J. M., Angelo, A. C., \& Britez, R. M. (2020). Crescimento de espécies arbóreas nativas em recuperação de área degradada no litoral do Paraná. Pesquisa Florestal Brasileira, 40. https://doi.org/10.4336/2020.pfb.40e201801680

Scherer, E. E., Nesi, C. N., \& Massotti, Z. (2010). Atributos químicos do solo influenciados por sucessivas aplicações de dejetos suínos em áreas agrícolas de Santa Catarina. Revista Brasileira de Ciência do Solo, 34, 1375-1383. https://doi.org/10.1590/S0100-06832010000400034

Silva, C. M. França, M. T., \& Oyamada, G. C. (2015). Características da suinocultura e os dejetos causados ao ambiente. Connection Line, 12, https://periodicos.univag.com.br/index.php/CONNECTIONLINE/article/view/199>.

Silva, J. B. G., Martinez, M. A., Matos, A. T., Oliveira, A. P. S., \& Silva, L. B. D. (2016). Concentração de metais em um solo adubado com água residuária da bovinocultura de leite. Revista Engenharia na Agricultura-Reveng, 24(4), 357-367.

Silveira, S. B., Neves, E. J. M., Carpanezzi, A. A., \& Britez, R. M. (2013). Avaliação silvicultural de Rapanea ferruginea e Citharexylum myrianthum plantadas em pastagens abandonadas. Pesquisa Florestal Brasileira, 33(73), 99-102. https://doi.org/10.4336/2013.pfb.33.73.410

Smanhotto, A., Sousa, A. P., Sampaio, S. C., Nóbrega, L. H. P., \& Prior, M. (2010). Cobre e zinco no material percolado e no solo com a aplicação de água residuária de suinocultura em solo cultivado com soja. Engenharia Agrícola, 30(2), 347-357. https://doi.org/10.1590/S0100-69162010000200017

Sorreano, M. C. M., Rodrigues, R. R., \& Boaretto, A. E. (2012). Guia de nutrição para espécies florestais nativas. São Paulo: Oficina de Textos. 260 p.

Souza, C. A. M., Oliveira, R. B., Filho, S. M., \& Lima, J. S. S. (2006). Crescimento em campo de espécies florestais em diferentes condições de adubações. Ciência Florestal, 16, 243-249. https://doi.org/10.5902/198050981905

Souza, J. A. R., \& Moreira, D. A. (2010). Efeitos do uso da água residuária da suinocultura na condutividade elétrica e hidráulica do solo. Engenharia Ambiental, 7, 134-143. < http://ferramentas.unipinhal.edu.br/engenhariaambiental/viewarticle.php?id=443\&layout=abstract .

Torres, F. S., Uhlmann, A., Pozzobon, M., Curcio, GR., Sevegnani, L., Braghirolli, F. L., Caglione, E., \& Volkmann, A. (2009). Recuperação de ambientes fluviais na Bacia Do Itajaí: Estabelecimento inicial de Citharexylum myrianthum e Schinus terebinthifolius sob diferentes espaçamentos e tipos de solos. Anais. IX Congresso de Ecologia do Brasil, 13 a 17 de Set. 2009, São Lourenço - MG. < https://ainfo.cnptia.embrapa.br/digital/bitstream/item/160301/1/638.pdf>.

United States Department of Agriculture - USDA (2016). Livestock and Poultry: World Markets and Trade. Foreign Agricultural Service. <http://www.fas.usda.gov/data/livestock-andpoultry-world-markets-and-trade>.

Vantroba, A. P., Bertolini, I. C., Sens, T. M. Z. G., Watzlawick, L. F., Schran, J. A., \& Pedroso, B. C. (2020). Características morfométricas e dendrocronológicas de Zanthoxylum rhoifolium Lam em fragmento de Floresta Ombrófila Mista Aluvial. Scientia Forestalis, 48 (127), e3338. https://doi.org/10.18671/scifor.v48n127.10

Vieira, A. R. R., Feistauer, D., \& Silva, V. P. (2009). Adaptação de espécies arbóreas nativas em um sistema agrossilvicultural, submetidas a extremos climáticos de geada na região de Florianópolis. Revista Árvore, 279 (5), 627-634. https://doi.org/10.1590/S0100-67622003000500005

Vogel, M. H. L., Schumacher, M. V., Storck, L., \& Witschoreck, R. (2005). Crescimento inicial de Pinus taeda relacionado a doses de N, P e K. Ciência Florestal, 15(2). https://doi.org/10.5902/198050981837 